\title{
REMOTE SENSING DATA AS BASIS FOR THE MODELLING AND REASSEMBLY OF DISMANTLED HERITAGE STRUCTURES
}

\author{
Stan Vincke ${ }^{1 *}$, Maarten Bassier ${ }^{1}$, Roberto de Lima Hernandez ${ }^{1}$, Ines Dejaeghere ${ }^{2}$, Kim Carbonez $^{2}$ and Maarten Vergauwen ${ }^{1}$ \\ ${ }^{1}$ Dept. of Civil Engineering, TC Construction - Geomatics, KU Leuven - Faculty of Engineering Technology, Ghent, Belgium \\ (stan.vincke,maarten.bassier,roberto.delimahernandez,maarten.vergauwen)@kuleuven.be \\ ${ }^{2}$ Monument Vandekerckhove NV \\ (Ines.Dejaeghere,Kim.Carbonez)@ monument-vandekerckhove.be
}

\author{
Commission II, WG II/8
}

KEY WORDS: Modelling, Restoration, Reassembly, Built Heritage, Remote Sensing

\begin{abstract}
:
Remote sensing techniques are invaluable for the documentation and preservation of built heritage. The techniques facilitate fast documentation of highly complex heritage structures with improved accuracies. Furthermore they improve the degree of detail substantially. This is extremely useful for the restoration of collapsed elements or the reassembly of dismantled structures. These entities are often challenging to puzzle back together. Moreover, the differential settlements of the elements over time heavily influence the relative position and orientation of the remaining pieces, further complicating the reconstruction. Digital modelling solutions with a 3D model of the current situation as take-off, are desperately needed by the industry to tackle the present obstacles. In this work, a framework is proposed that facilitates a more accurate reassembly of dismantled heritage elements. It consists of three major phases starting with the accurate recording of the current situation as well as the preserved components. Subsequently, the new design is dititally modelled, reducing the necessary time for the reassembly of the structure, which is the last step in the rebuilding workflow. The presented framework allows for an efficient and comprehensible reconstruction of the structure. A key aspect in the approach is the detection of missing components and the estimation of their dimensions for the production of accurate replicas. The potential is showcased by means of two case studies on the reassembly of flying buttresses and rib vaults of the Saint-James church in Leuven, Belgium, which is currently undergoing major stabilisation works. The presented approach allows heritage experts to gain better oversight over their reassembly project and work more efficiently.
\end{abstract}

\section{INTRODUCTION}

Documentation is paramount in the field of built cultural heritage. Thoroughly monitoring and documenting the encountered deflections is an important aspect in the conservation of heritage (Remondino, 2011). Also for the restoration of heritage structures, information on the previous state is essential (Tapete et al., 2013). When documentation is available of what the former situation looked like, collapsed heritage entities can be restored either to their original state or to a slightly modified state, for instance using internal reinforcements. Also heritage that is dismantled can be reassembled in a later stage according to the former reality.

Digitally modelling the new design has numerous advantages. Modifications in the new design inevitably are necessary when the previous structure collapsed but also when elements were dismantled for safety measures for instance. These modifications can easily be executed in digital models. Furthermore it allows for taking into account the changed building conditions compared to when the heritage structure was originally built. Various software provide tools to design a new structural entity based on the $3 \mathrm{D}$ model representing the remaining parts of the heritage structure (figure 1). To this end, remote sensing techniques are frequently used to record the current situation in a high degree of detail.

Heritage restoration often requires the reuse of original materials (Yaka çetin et al., 2012). The intact structure with all the original components in-place often is documented poorly or not documented at all. Furthermore, several elements of the structure

\footnotetext{
${ }^{*}$ Corresponding author
}

might be missing or broken. The digital models of the preserved building components allow for digitally puzzling the fragments together. This offers a significant advantage over the manual reassembly since it reveals missing elements much faster, allowing for the sculpturing of new gap-filling elements for instance. Furthermore, digital puzzling is less labour intensive opposed to its manual counterpart. Moreover, the approach is less confusing since all component models are digitally named.

The emphasis of this work is on the modelling of dismantled structural heritage elements and how remote sensing data as a basis can be beneficial in this process. This is researched by means of two case studies where the rebuilding pipeline is discussed from recording the current situation to the reassembly of the heritage structures.

The remainder of this work is structured as follows. In section 2 . the history of the church is discussed as well as related work regarding documentation, modelling and restoration of cultural heritage. Subsequently, the methodology is presented in section 3 . The two cases of the reassembly of the flying buttresses and rib vaults are presented in section 4 . and 5. Finally, the conclusions are presented in section 6 .

\section{BACKGROUND AND RELATED WORK}

The Saint-James church, providing two case studies in this work, dates back to the early 13th century. Differential settlements occurred due to excessive surcharges by adaptations in the 16th centuries and the location originally being a swamp. In 1963, the church was closed for public access because of safety precautions (Smars et al., 2006, Schueremans et al., 2007). In order to 
The International Archives of the Photogrammetry, Remote Sensing and Spatial Information Sciences, Volume XLII-2/W15, 2019 27th CIPA International Symposium "Documenting the past for a better future", 1-5 September 2019, Ávila, Spain

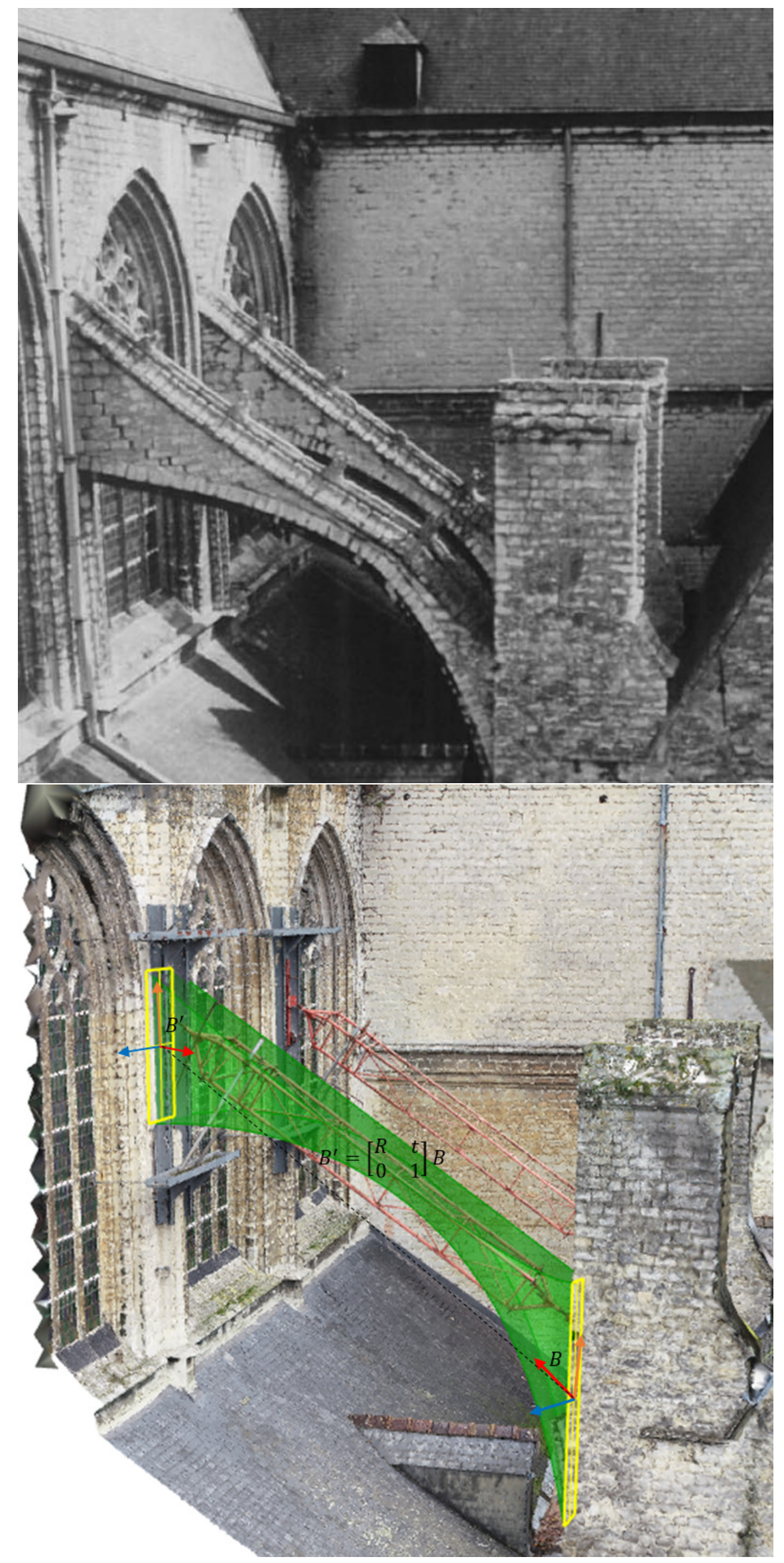

Figure 1: The upper figure shows the former flying buttresses. The existing configuration of the remaining heritage structure is shown in the bottom figure. The 3D model provides the opportunity to calculate the transformation parameters between the two surfaces $\mathrm{B}$ and B', which is useful for the new design, adapted to the current situation. 
have access to all structural parts and to stabilise the church, a substantial part of the masonry was removed, including the masonry rib vaults of the side naves and the flying buttresses on the outside. These structures were replaced by provisional support structures (figure 1 (bottom)). Furthermore, upon dismantling, all original stones were numbered and photographed, while still in-place, for later reference (figure 2a) (Smars et al., 2006).

Also in related works, large effort has been put in restoring and reassembling dismantled or broken heritage structures. Various work has been focussing on the reassembly of heritage fragments. Up until recently this work was mostly carried out manually by heritage experts. The works of Adan et al., Gregor et al., and Papaioannou et al. focus on digitally reassembling broken artefacts (Adán et al., 2012, Gregor et al., 2014, Papaioannou et al., 2017). Brown et al. present a similar paper but focus on the reassembly of fresco paintings (Brown et al., 2012). It is also possible to enhance the reconstructed heritage elements with additional components, such as Arbace et al. have done in their work (Arbace et al., 2013). They use an underlying support structure for the renovation of a damaged statue. Moreover, they also include digital models in the reconstruction pipeline.

All the former mentioned works share a common prerequisite: the need for 3D models of preserved elements, similar as in our work. Remote sensing techniques such as laser scanning and photogrammetry serve this purpose exceptionally well (Yastikli, 2007). The resulting models, but also derived data such as orthophotos, serve as excellent take-offs in restoration and reassembly processes. In contrast with the 3D models of small artefacts, also larger structures are possible to capture with these techniques. Various works successfully record and virtually reconstruct the current situation of heritage buildings in 3D. Remote sensing techniques can be used for the monitoring of structural deformations in heritage buildings (Tapete et al., 2013, SuziedelyteVisockiene et al., 2015). Furthermore, the models can also be used for structural analysis purposes such as discussed by Armesto et al. and Bassier et al. among others (Armesto et al., 2010, Bassier et al., 2019).

This work focusses on the reassembly of larger heritage structures such as flying buttresses and rib vaults, presented in the case studies. By following a digital approach and using the input information of remote sensing-based models, the design and reassembly of heritage structures can be executed more efficiently.

\section{METHODOLOGY}

In this paper a framework is presented for the reassembly of dismantled heritage structures. It consists of four major consecutive steps, namely the acquisition of $3 \mathrm{D}$ representations of the current situation, the capturing of preserved building components, the modelling process and finally the rebuilding phase. These steps are discussed in the following paragraphs.

Capturing current situation The accurate 3D representation of the current situation of the remaining parts of the heritage structure is vital for the modelling process. Therefore remote sensing techniques are employed to satisfy the accuracy requirements on multiple levels. First at all, the representation has to be globally accurate, correctly describing the respective positions of the opposing bases. Furthermore the representation is also required to describe the remaining bases of the dismantled elements in a high degree of detail, since new keystones are modelled based on this information. For the creation of this accurate model, two remote sensing techniques are used. Laser scanning provides the accurate relative positions of different remaining elements. On the other hand photogrammetry is used, both based on terrestrial as well as aerial imagery, for the detailed description of the remaining bases of the dismantled elements.

Capturing preserved elements The representations of the original building components are a second valuable input for the new design of the dismantled heritage entities. In both case studies the original materials were preserved after dismantlement. Monument Vandekerckhove, a Belgian construction company specialised in restoration and renovation projects, is responsible for the modelling and reassembly of the dismantled structures in both case studies. Manual measurements of the most significant features currently serve as basis for the preserved element models. Obviously, this documentation has a rather limited degree of detail. By using a structured light hand scanner, following a recording approach as described in previous work (Bassier et al., 2018), the current low degree of detail is substantially increased. Furthermore, because of the similar shapes of the stones (figure 2a) only a representative subset needs to be captured. More complex forms such as figure $2 b$ and $2 c$, are captured substantially easier and more accurate with this technique. Subsequent to their dismantlement, the original stones typically are cleaned and disposed from the remaining mortar rests. This provides the ideal occasion for capturing these components. Throughout the rest of the design process up until the final reassembly, the stones can be stored. This way these entities are only moved once, hence better preserving their sometimes delicate state.

Modelling In the modelling phase the results of the two former methodology phases are combined. The digital design of the new structure starts from the current situation. Over time differential settlements can have occurred, such as in both case studies, making it impossible to restore the structure as an exact replica of the original structure. The accurate remote sensing model of the current situation allows for creating 3D splines between the opposing surfaces. By digitally modelling profiled extrusions along the spline a first draft of the new restored element is created. Subsequently, by placing the design in-place in the $3 \mathrm{D}$ representations of the remaining parts, gaps, misalignments and other errors are revealed. As this is performed digitally, the design can easily be updated appropriately. By repeatedly going through this process, a final design is formed.

In the second stage of the modelling process, the internal elements of the structure are considered. The captured elements are fit into the new design. This allows restorers to check for missing elements. It often occurs that some of the original elements are missing or in a non-acceptable state. The digital design in combination with the digital models of the preserved elements allows restorers to sculpture new replica elements to fill the gaps. Moreover, since the current state can have changed compared to the situation the elements were originally designed for, the digital approach provides us the opportunity to check if the form of original elements is suitable for the updated design or if it is required to slightly change their form. Furthermore, extra requirements such as internal reinforcements, can be accounted for as well during the modelling.

Reassembly The detail and accuracy of the 3D model and the digital design allow for reassembling the new structure off-site, either partially or fully. This would yield the advantage of reassembling the components under far better circumstances than at the in-place reconstruction. However, although in theory this is possible, in reality this approach mostly is not realised. Certainly if complexity levels rise, such as in both case studies, placing the reassembled structures correctly in place, is mostly infeasible. Nevertheless the proposed digital modelling approach yields 


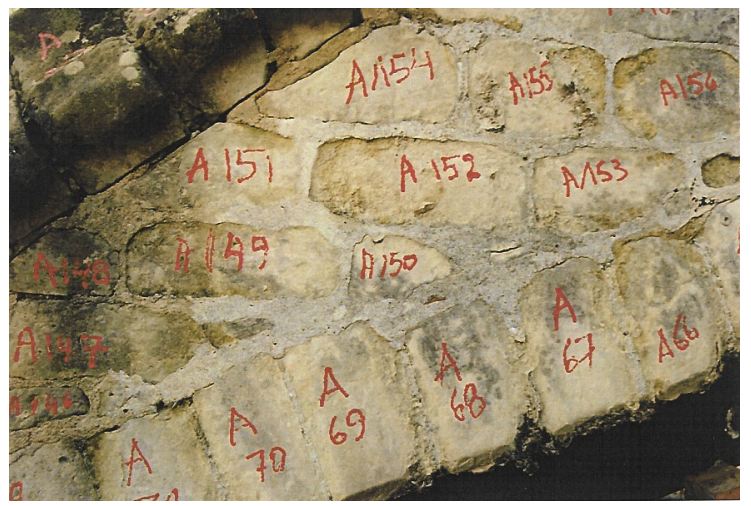

(a)

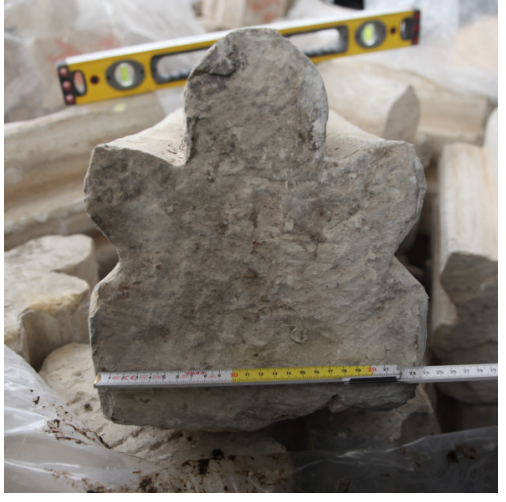

(b)

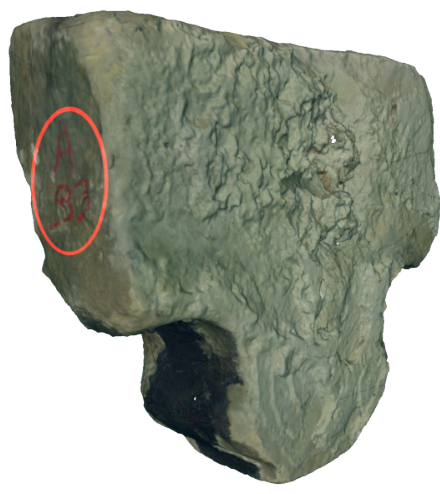

(c)

Figure 2: Examples of stones captured by a structured light hand scanner, ranging from simple to complex (a) photograph of the numbered cubic-shaped stones upon dismantlement (b) symmetrical rib vault stone (c) numbered flying buttress stone (circled left upper surface)

advantages over the current more manual approaches in this stage of the reassembly process as well. No unexpected placement conditions can arise for instance. Since the remaining parts are captured in high detail, elements can be modelled on beforehand such that they exactly fit under the current changed circumstances in contrast to the original situation.

\section{CASE STUDY 1: FLYING BUTTRESSES}

Typical for the Gothic style of the Saint-James church is the presence of flying buttresses. These elements were added to the church between 1534 and 1535 (Schueremans et al., 2007). Due to differential settlements, the stability of the flying buttresses was severely endangered. Therefore the structures were dismantled and replaced by provisional tie-rods in the year 2000 since small additional displacements could have led to the collapse of the elements (Smars et al., 2006, Verstrynge et al., 2012). Currently, the church is stabilised by micro poles as part of the ongoing restoration. This allows for reassembling the flying buttresses. In the following paragraphs the different phases of the rebuilding pipeline are discussed in line with the phases described in the methodology.

Capturing current situation Manual measurements of the desired dimensions would have been hard to impossible due to the challenging accessibility of the site. The opposing remaining bases lie approximately five metres apart without a direct physical link between them except for a hard to capture steel support structure and a roof, approximately three metres under the bases of the buttresses. For the new design of the structures ${ }^{1}$, a high quality $3 \mathrm{D}$ reconstruction is required. Therefore we digitally reconstruct the scene in 3D using an integration of two remote sensing techniques, namely laser scanning and photogrammetry, fulfilling the accuracy requirements on different levels.

The challenging circumstances rule out most other digital reconstruction techniques than laser scanning. Therefore, a Leica BLK laser scanner is used to capture the present situation. For each side of the church four scans were recorded, of which two are made from a position on a man lift and the other two from the chapel windows located above the flying buttresses (upper side of figure 1 (top)). Secondly, we use photogrammetry to reconstruct the scene with a much higher degree of detail, compared to the achievable results of the laser scanner. As a result of the inaccessibility of the scene, a recording workflow only using a hand-held

\footnotetext{
${ }^{1}$ Executed by Monument Vandekerckhove
}

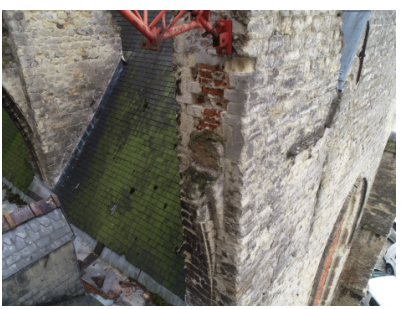

(a)

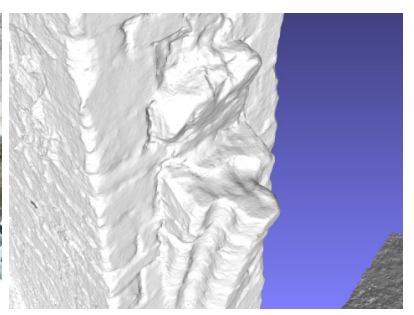

(b)
Figure 3: The highly detailed 3D model of the scene was obtained by employing a UAV (a) example of a full 20MP image, showing the detail of the captured texture (b) part of the highly detailed resulting mesh model

camera is infeasible. Therefore we additionally use an Unmanned Aerial Vehicle (UAV) to record the scene. By carefully navigating the UAV under, over and in between the two support structures, facing both the main nave and the flying buttresses piers, the remaining bases are recorded in very high detail as shown in figure $3 \mathrm{a}$. The flying buttresses at the northern and southern side of the church are captured with 384 and 279 images respectively. Apart from the high texture quality, this approach also delivers a highly detailed mesh of the remaining attachments (figure $3 b$ ) which serves as invaluable input for the modelling of the crucial keystones.

The entire project is split into two sub-projects, one for the two northern and one for the two southern flying buttresses. The captured laser scans are registered using the Leica Cyclone Register 360 software (Leica, 2019). Subsequently, upon importing the laser scan data into the photogrammetric software RealityCapture (CapturingReality, 2019), the laser scan registration is frozen so no alterations can be applied during further processing. This ensures that the metric quality of the laser scanning datasets is preserved in the end product. Via the available colour information in the scans, a direct link is calculated between the UAV imagery and the scans. The processing results in two meshes with three million faces each and ten $4 \mathrm{k}$ texture maps resulting in a texel size of $2 \mathrm{~mm}$, hence providing the required high resolution.

One of the results is shown in figure 4. It shows the displacement dimensions overlaid on an orthophoto of one of the flying buttresses. The displacement in the horizontal Y-direction is $34,8 \mathrm{~cm}$. Presumably this is the result of differential settlements in combination with an inaccurate former building process. Also in the Xdirection displacements might have occurred over time, however, as no original building plans are available and the displacement 
cannot be deducted solely from the current situation, this offset remains unknown. Also for the vertical displacement this is the case. It is a fact that differential settlements have occurred, however, the degree to which the two bases have differentially settled is unknown. Aside from all displacements, also rotational effects occurred. Between the two depicted remaining bases of one of the flying buttresses, the relative rotation is 1,37 degrees.

Capturing preserved stone elements One of the prerequisites for the modelling of the new flying buttresses is the recording of geometric data of the original stone elements. Because of the similarity between the stones only a representative subset of the stones is captured using the Einscan Pro Plus structured light hand scanner. For each stone archetype a 3D model is created. The flying buttresses were dismantled in the year 2000 and the elements were stored inside the church, closed for public. However, for the restoration, the original stones are required to be cleaned and undone from remaining mortar rests. This provides the perfect opportunity to scan them and yields the advantage that the elements only are moved and handled once, hence better preserving the sometimes delicate state.

The actual scanning time takes approximately three minutes, depending on the complexity and size of the element. In a subsequent phase the scan results are processed to a coloured point cloud and textured mesh. This approximately takes another three minutes, hence resulting in a total six minutes for the whole process on average. The 3D meshes of the building components for the new structure consist of many faces, ranging from 300.000 for simpler geometries (figure 2a) to 600.000 faces and up for more complex stones (figure $2 \mathrm{~b}$ and $2 \mathrm{c}$ ).

The presented capturing approach produces models with a substantially higher degree of detail in comparison with manually measuring the elements. Although for the simpler, bar-shaped forms of stones, the capturing approach does not yield substantially better or more usable results, for complex geometries this is the case. In this case study the capturing of the preserved stone elements therefore is considered as proof of concept, yielding the most added value for elements with a more complicated geometry.

Modelling of the new flying buttresses In a first stage of creating a new design ${ }^{2}$ of the flying buttresses, the current situation, captured by remote sensing, is evaluated. By means of a combination of the 3D models and extracted orthophotos of the current situation, three-dimensional splines are designed that connect the two remaining bases (figure 5a). This is done both for the bottom and top row of the arch since these stone rows form the main force-bearing structures. Subsequently, the profiles of the bottom and top stones, obtained via the structured light scanner, are extruded along these splines (figure $5 \mathrm{~b}$ and $5 \mathrm{c}$ ). By digitally modelling, it is possible to check for gaps or misalignments. Also the best-fitting keystones at the beginning and end of the arches are determined this way.

A second phase consists of assessing the necessary components to form the flying buttress. The design is elaborated by filling up the space between the two stone rows with bar-shaped stones. This step is performed in 2D. As a result a plan such as depicted in figure $5 \mathrm{~d}$ is obtained. Next, the plan is enhanced with several indications for each stone. First at all the names are indicated. These are partly based on the photographs of the physically marked stones, taken upon dismantlement. Moreover, it is indicated whether or not the stones are still present or are unusable because of their bad state. Because of the altered situation

\footnotetext{
${ }^{2}$ Executed by Monument Vandekerckhove
}

compared to the original designs, also adaptations to the existing stones were foreseen. However, the deviations from the desirable form are rather small such that they can be counteracted by the layer of mortar in-between the stones, hence avoiding the need for reshaping stone elements.

Reconstruction of the new flying buttresses The plans, created in the former step, excellently keep track of elements that need to be replicated to fill up the gaps left behind by missing or deteriorated stones. These new elements, such as in figure $6 \mathrm{~d}$, are created based on the scans of the archetypes of the used elements. The raw stone shapes from the quarry are first roughly cut and sculptured by machinery and subsequently are handcrafted further by restorer specialists to their final desired form.

As mentioned in the methodology, the scene is too complex for an off-site reconstruction. Although this was originally proposed in the tender offer, the size and form of the flying buttresses is too complicated for a solid, correct placement of the rigid stone structure. In contrast to the stone arches, however, it is possible to place a pre-fabricated wooden formwork (figure 6a) under the remaining bases for supporting the arch elements upon reconstruction. The creation of such perfectly fitting prefabricated formwork is possible due to the highly accurate models of the current situation, showing the advantages of the presented approach. The next step consists of placing the bottom row of force-bearing stones (figure 6b). The physically numbered stones are placed according to their plan number. Subsequently, the inner stones are placed following the same approach, forming a planar shaped upper surface (figure $6 \mathrm{c}$ ). This is covered by the top row of stones, forming the second force-bearing part of the new flying buttress structure (figure 6d).

\section{CASE STUDY 2: RIB VAULTS}

The rib vaults were originally added to the church between 1305 1317 , replacing the former wooden structure. In the period 14851487 , the masonry vaults were reconstructed after cracks appeared in the former structure due to differential settlements. The unstable underground of the church in combination with extra loads induced by heightening the original construction, aggravated the differential settlements even further up until recently. Shortly after closing the church for public access in 1963 for safety reasons, the masonry rib vaults were dismantled in the period of 19651971 (Schueremans et al., 2007).

Since the church is currently stabilised by means of micro-poles, the reconstruction of the rib vaults becomes possible. This forms the subject of the second case study. In the remainder of this section, the restoration of the rib vaults and more specifically the ribs themselves, forming the basis of the structure, is discussed. The rib elements are restored following the approach presented in the methodology. Since the method is rather similar to the other presented case study focussing on the restoration of the flying buttresses, this case study is discussed more briefly.

Capturing current situation The current situation of the interior of the church and the bases for the rib vaults is recorded using laser scanning. For other restoration purposes all of the church's main pillars were surrounded by scaffolds. These pose an environment challenging to record and made it impossible to use photogrammetry as second technique. A highly accurate Leica P30 laser scanner was used to capture a total of 17 high resolution scans for the rib vaults above the two side naves. The scan results were further processed and finally underwent a large cleaning phase to remove all scaffold structures in the recorded data. 


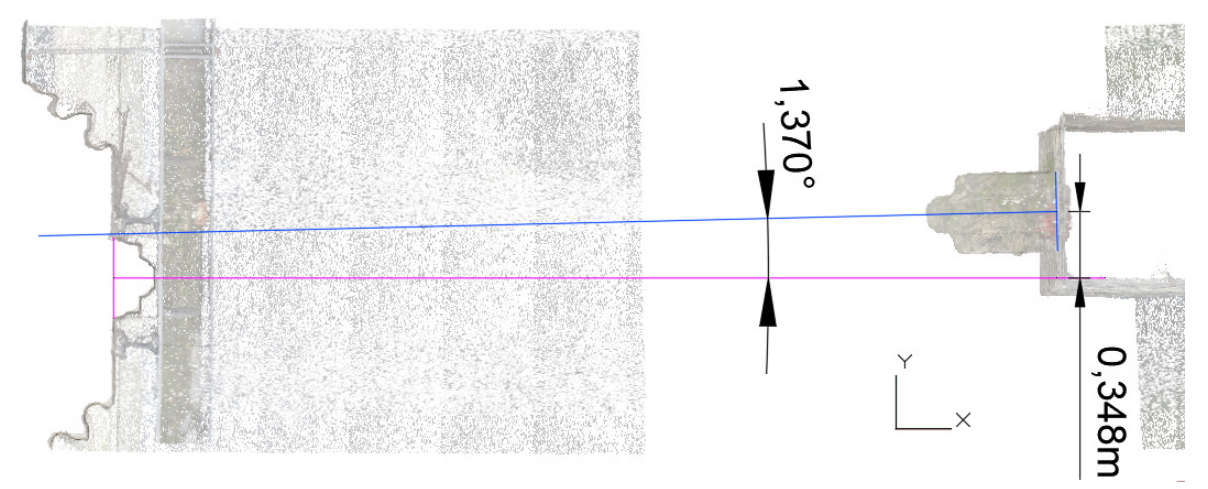

Figure 4: Top-view orthophoto depicting the displacements of one of the flying buttresses. The horizontal displacement in the Ydirection is $34,8 \mathrm{~cm}$. The two bases are also rotated for 1,37 degrees relative to each other.

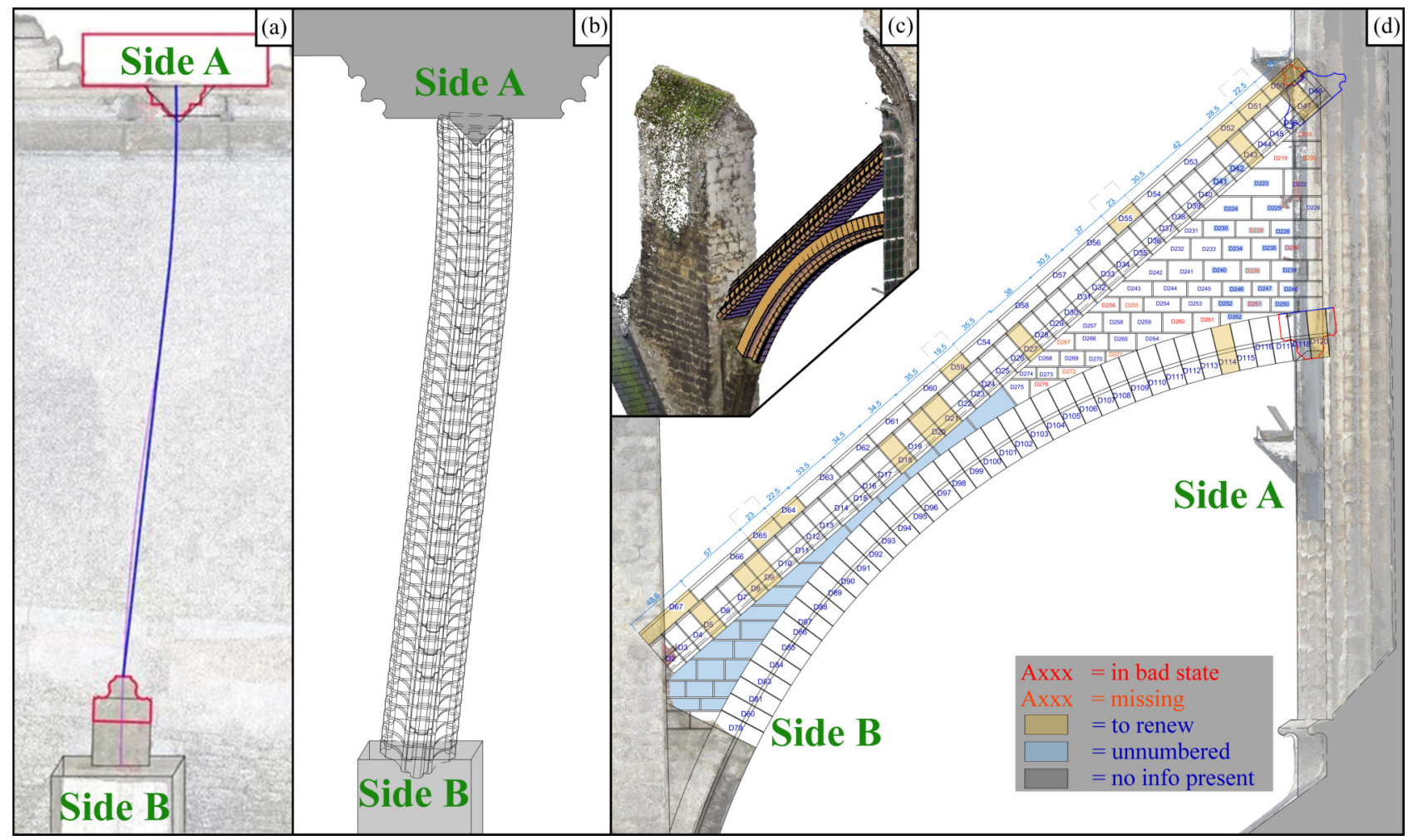

Figure 5: Extraction of some of the plan elements for the new design of one of the flying buttresses depicting the design workflow. (a) 3D spline creation (b) extrusion of profile along spline (c) 3D visualisation of the extruded top and bottom row of the arch (d) 2D plan with indications for each stone.

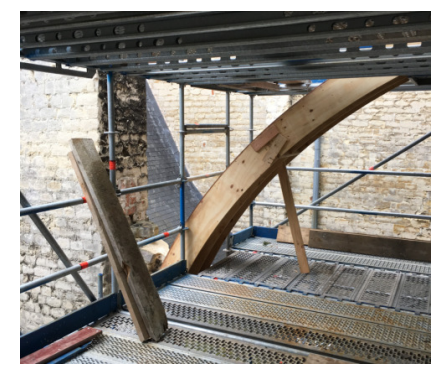

(a)

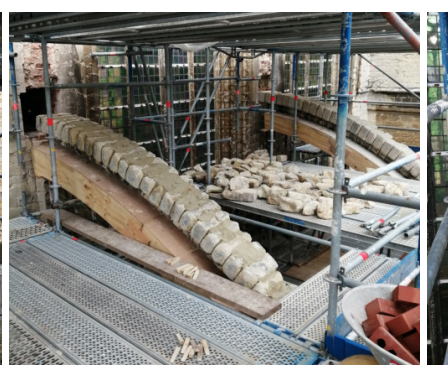

(b)

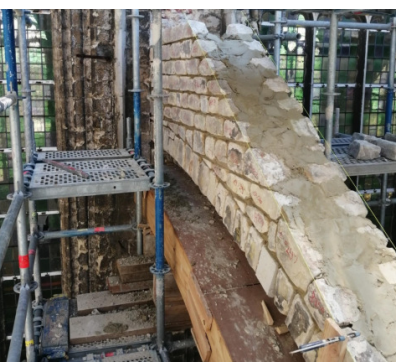

(c)

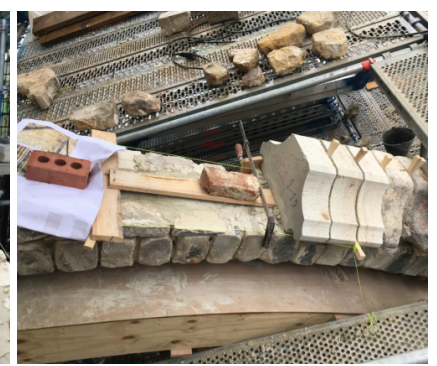

(d)

Figure 6: Figure depicting the subsequent steps in the reconstruction process of the flying buttresses (a) supporting formwork (b) first row of force-bearing stone elements (c) inner stone placement (d) top row force-bearing elements placement, note the replica stones substituting missing elements 

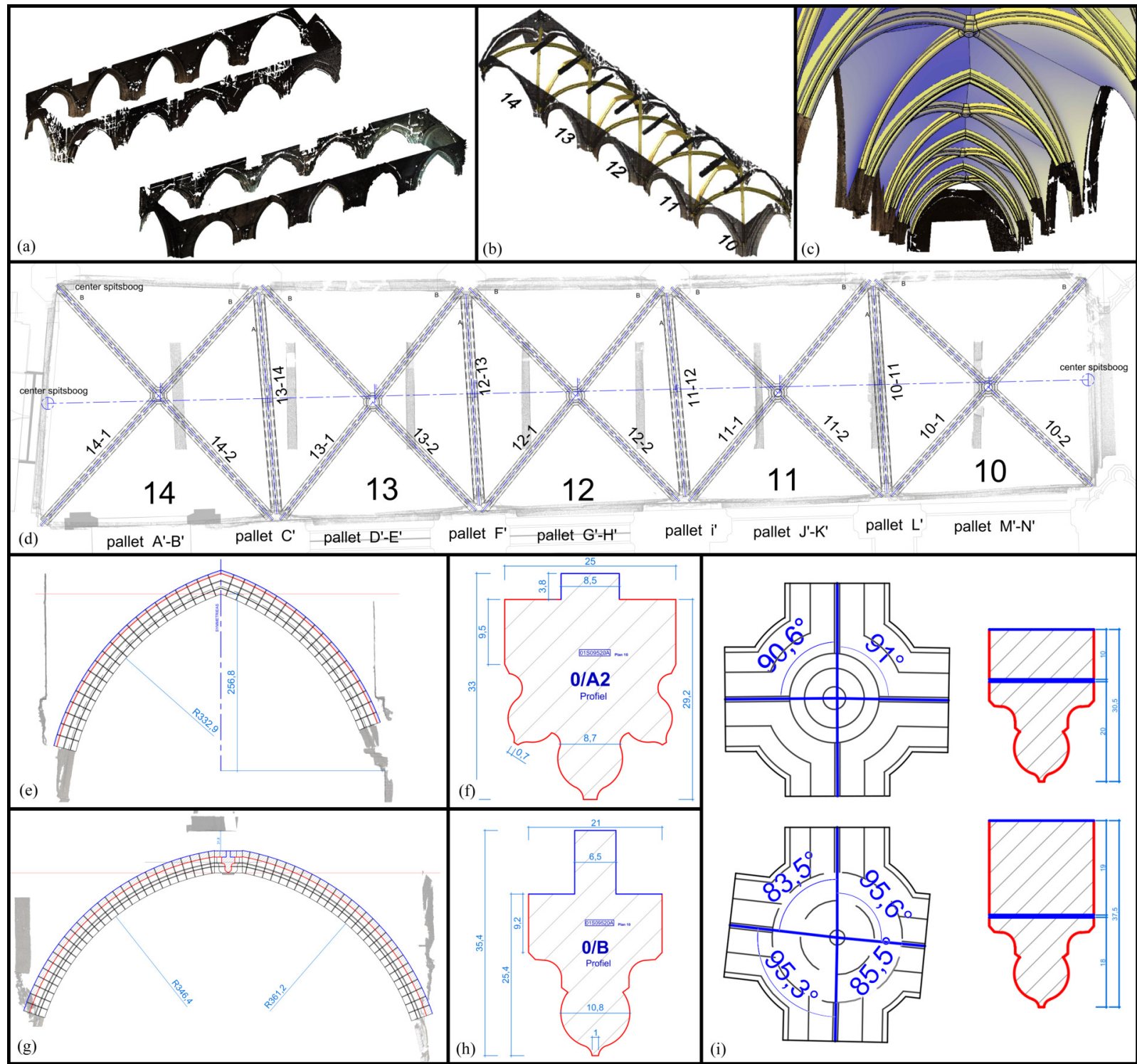

Figure 7: Subset of plan elements for the reconstruction of the vaults' ribs. (a) Laser scanning result of the recorded current situation (b $\&$ c) 3D visualisations of the modelled rib vaults (d) 2D plan, note the asymmetrical characteristics of the structure due to differential settlements (e) plan for one of the pointed arches (f) stone profile for the pointed arches (g) plan for one of the vault ribs, note the different radii of the arch parts (h) stone profile for the vault ribs (i) designs for two replicas of rib vault centre keystones, note the large deviations from the ideally perpendicular 90 degrees intersections.

This resulted in two point clouds, one for each side nave, of each approximately 8 million points after reducing the densities (figure 7a).

Capturing preserved stone elements In a second phase the archetypes of the preserved stone elements are captured. For each rib section the stones share the same shape and therefore are not numbered. However, scanning several elements was still useful in this case for the creation of the profile of the stones (figure $7 \mathrm{f}$ and $7 \mathrm{~h}$ ). The profile depicted in figure $7 \mathrm{f}$ is based on a slice of the structured light scan results of the stone depicted in figure $2 b$.

Modelling of the new rib vaults Due to the differential settlements, the current situation differs substantially from the original state (figure $7 \mathrm{~d}$ ). Over time, the rib vaults have been reconstructed several times. Due to differential settlements, the central keystones strayed further and further from the presumably symmetrical and perpendicular original shape. Several of these centrepieces are preserved and the missing ones are replicated (figure 7i). The known shape of these pieces in combination with the accurate 3D model of the current situation, results in a good insight in the former shapes of the rib vaults. Based on this, 3D splines are created between the opposing bases. The created profiles are subsequently extruded along these. This way the final models are formed for the ribs (figure $7 \mathrm{~b}$ and $7 \mathrm{c}$ ) and $2 \mathrm{D}$ plans are extracted (figure $7 \mathrm{e}$ and $7 \mathrm{~g})^{3}$. Compared to the other case study, the modelling process is easier in this case since there is only one chain of stones. Furthermore, the regular shape of each arch segment does not necessitate numbered stones.

Reconstruction of the new rib vaults The reconstruction of the new rib vaults has not been executed yet at the time of writing this work. The elements are not yet accessible due to scaffolding structures for the restoration of other parts in the interior of the church.

\footnotetext{
${ }^{3}$ Executed by Monument Vandekerckhove
} 


\section{CONCLUSION}

The input of remote sensing data in the design process for the restoration or reassembly of dismantled heritage structures yields substantial advantages over current approaches. The techniques enable a fast and accurate capturing of the current configuration of the sometimes complex remaining structures. Moreover, the differential settlements that happened over time are recorded and can be accounted for. The input of a highly detailed model of the present situation in combination with models accurately describing the shapes of the preserved elements, substantially reduce the complexity of puzzling the dismantled structures back together.

In this work, a framework is proposed that unites the technological advances in 3D modelling with the reassembly of dismantled heritage elements. Subsequent to the capturing of the current situation and the recording of preserved elements, a digital model is created. Consequently, this reduces the time necessary for the reconstruction of the structure and, because of the digitisation, allows for working more remotely. Furthermore, the reassembly complexity is reduced. Moreover, the framework also facilitates other opportunities such as the detection of missing components and the determination of the required dimensions of replica elements to fill up the gaps.

The potential of the method is showcased in two case studies in the Saint-James church in Leuven, Belgium. The church recently underwent major stabilisation work, necessary due to differential settlements. The presented approach was followed in the reassembly projects of the flying buttresses and rib vaults and has been proven successful. It allows heritage experts to gain better oversight over their reassembly project and work more efficiently.

\section{ACKNOWLEDGEMENTS}

This project has received funding from the FWO research foundation (under FWO PhD SB fellowship 1S11218N) and the Geomatics research group of the Department of Civil Engineering, TC Construction at the KU Leuven in Belgium. The authors also would like to thank Monument Vandekerckhove for the cooperation.

\section{REFERENCES}

Adán, A., Salamanca, S. and Merchán, P., 2012. A hybrid humancomputer approach for recovering incomplete cultural heritage pieces. Computers and Graphics (Pergamon) 36(1), pp. 1-15.

Arbace, L., Sonnino, E., Callieri, M., Dellepiane, M., Fabbri, M., Iaccarino Idelson, A. and Scopigno, R., 2013. Innovative uses of $3 \mathrm{D}$ digital technologies to assist the restoration of a fragmented terracotta statue. Journal of Cultural Heritage 14(4), pp. 332345 .

Armesto, J., Roca-Pardiñas, J., Lorenzo, H. and Arias, P., 2010. Modelling masonry arches shape using terrestrial laser scanning data and nonparametric methods. Engineering Structures 32(2), pp. 607-615.

Bassier, M., Hardy, G., Bejarano-Urrego, L., Drougkas, A., Verstrynge, E., Van Balen, K. and Vergauwen, M., 2019. Semiautomated Creation of Accurate FE Meshes of Heritage Masonry Walls from Point Cloud Data. 18, pp. 305-314.

Bassier, M., Vincke, S., Hernandez, R. D. L. and Vergauwen, M., 2018. An Overview of Innovative Heritage Deliverables Based on Remote Sensing Techniques. Remote Sensing.
Brown, B., Laken, L., Dutré, P., Van Gool, L., Rusinkiewicz, S. and Weyrich, T., 2012. Tools for Virtual Reassembly of Fresco Fragments. International Journal of Heritage in the Digital Era 1(2), pp. 313-329.

\section{CapturingReality, 2019. RealityCapture.}

Gregor, R., Sipiran, I., Papaioannou, G., Schreck, T., Andreadis, A. and Mavridis, P., 2014. Towards Automated 3D Reconstruction of Defective Cultural Heritage Objects. pp. 135-144.

Leica, 2019. Cyclone Register 360.

Papaioannou, G., Schreck, T., Andreadis, A., Mavridis, P., Gregor, R., Sipiran, I. and Vardis, K., 2017. From Reassembly to Object Completion. Journal on Computing and Cultural Heritage 10(2), pp. 1-22.

Remondino, F., 2011. Heritage recording and 3D modeling with photogrammetry and 3D scanning. Remote Sensing 3(6), pp. 1104-1138.

Schueremans, L., Van Balen, K., Brosens, K., Van Gemert, D. and Smars, P., 2007. Church of Saint-James at leuven: Structural Assessment and Consolidation Measures. International Journal of Architectural Heritage 1(1), pp. 82-107.

Smars, P., Schueremans, L. and Van Balen, K., 2006. Monitoring the dismantlement of four flying buttresses. Structural Analysis of Historical Constructions, New Delhi, India 3, pp. 1421-1428.

Suziedelyte-Visockiene, J., Bagdziunaite, R., Malys, N. and Maliene, V., 2015. Close-Range Photogrammetry Enables Documentation of Environment-Induced Deformation of Architectural Heritage. Environmental Engineering and Management Journal 14(6), pp. 1371-1381.

Tapete, D., Casagli, N., Luzi, G., Fanti, R., Gigli, G. and Leva, D., 2013. Integrating radar and laser-based remote sensing techniques for monitoring structural deformation of archaeological monuments. Journal of Archaeological Science 40(1), pp. 176189.

Verstrynge, E., Schueremans, L. and Smars, P., 2012. Controlled intervention: Monitoring the dismantlement and reconstruction of the flying buttresses of two Gothic churches. International Journal of Architectural Heritage 6(6), pp. 689-708.

Yaka çetin, F., Ipekoglu, B. and Laroche, D., 2012. Reconstruction of archaeological sites: Principles practice and evaluation. International Journal of Architectural Heritage 6(5), pp. 579603.

Yastikli, N., 2007. Documentation of cultural heritage using digital photogrammetry and laser scanning. Journal of Cultural Heritage 8(4), pp. 423-427. 